\title{
The nucleosynthesis of heavy elements in Stars: the key isotope ${ }^{25} \mathrm{Mg}$
}

C. Massimi ${ }^{1,2, a}$, P. Koehler ${ }^{3}$, S. Kopecky ${ }^{4}$, F. Mingrone ${ }^{1,2}$, P. Schillebeeckx ${ }^{4}$, G. Vannini ${ }^{1,2}$, S. Altstadt ${ }^{5}$, J. Andrzejewski ${ }^{6}$, L. Audouin ${ }^{7}$, M. Barbagallo ${ }^{8}$, V. Bécares ${ }^{9}$, F. Bečváŕr ${ }^{10}$, F Belloni ${ }^{11}$, E. Berthoumieux ${ }^{11,12}$, J. Billowes ${ }^{13}$, D. Bosnar ${ }^{14}$, M. Brugger ${ }^{12}$, M. Calviani ${ }^{12}$, F. Calviño ${ }^{15}$, D. Cano-Ott ${ }^{9}$, C. Carrapiço ${ }^{16}$, F. Cerutti ${ }^{12}$, E. Chiaveri ${ }^{12,11}$, M. Chin $^{12}$, N. Colonna ${ }^{8}$, G. Cortés ${ }^{15}$, M.A. Cortés-Giraldo ${ }^{17}$, M. Diakaki ${ }^{18}$, C. Domingo-Pardo ${ }^{19}$, I. Duran ${ }^{20}$, R. Dressler ${ }^{21}$, C. Eleftheriadis ${ }^{22}$, A. Ferrari ${ }^{12}$, K. Fraval ${ }^{11}$, S. Ganesan ${ }^{23}$, A.R. García ${ }^{9}$, G. Giubrone ${ }^{19}$, I.F. Gonçalves ${ }^{16}$, E. González-Romero ${ }^{9}$, E. Griesmayer ${ }^{24}$, C. Guerrero ${ }^{12}$, F. Gunsing ${ }^{11}$, A. Hernández-Prieto ${ }^{12,}$, D.G. Jenkins ${ }^{25}$, E. Jericha ${ }^{24}$, Y. Kadi ${ }^{12}$, F. Käppeler ${ }^{26}$, D. Karadimos ${ }^{18}$, N. Kivel ${ }^{20}$, M. Krtička ${ }^{10}$, J. Kroll ${ }^{10}$, C. Lampoudis ${ }^{11}$, C. Langer ${ }^{5}$, E. Leal-Cidoncha ${ }^{20}$, C. Lederer ${ }^{27}$, H. Leeb ${ }^{24}$, L.S. Leong ${ }^{7}$, R. Losito ${ }^{12}$, A. Mallick ${ }^{23}$, A. Manousos ${ }^{22}$, J. Marganiec ${ }^{6}$, T. Martínez ${ }^{9}$, P.F. Mastinu ${ }^{28}$, M. Mastromarco ${ }^{8}$, E. Mendoza ${ }^{9}$, A. Mengoni ${ }^{29}$, P.M. Milazzo ${ }^{30}$, M. Mirea ${ }^{31}$, W. Mondalaers ${ }^{4}$, C. Paradela ${ }^{20}$, A. Pavlik ${ }^{27}$, J. Perkowski ${ }^{6}$, A. Plompen ${ }^{4}$, J. Praena ${ }^{17}$, J.M. Quesada ${ }^{17}$, T. Rauscher ${ }^{32}$, R. Reifarth ${ }^{5}$, A. Riego ${ }^{32}$, M.S. Robles ${ }^{20}$, C. Rubbia ${ }^{12}$, M. SabatéGilarte $^{17}$, R. Sarmento ${ }^{16}$, A. Saxena ${ }^{23}$, S. Schmidt ${ }^{5}$, D. Schumann ${ }^{21}$, G. Tagliente ${ }^{8}$, J.L. Tain ${ }^{19}$, D. Tarrío ${ }^{20}$, L. Tassan-Got ${ }^{7}$, A. Tsinganis ${ }^{12}$, S. Valenta ${ }^{10}$, V. Variale ${ }^{8}$, P. Vaz $^{16}$, A. Ventura ${ }^{29}$, M.J. Vermeulen ${ }^{25}$, V. Vlachoudis ${ }^{12}$, R. Vlastou ${ }^{18}$, A. Wallner ${ }^{27}$, T. Ware ${ }^{13}$, M. Weigand ${ }^{5}$, C. Weiß ${ }^{24}$, T. Wright ${ }^{13}$, and P. Žugec ${ }^{14}$

${ }^{1}$ Dipartimento di Fisica e Astronomia, Università di Bologna, I-40136, Bologna, Italy

${ }^{2}$ Istituto Nazionale di Fisica Nazionale - Sezione di Bologna, I-40137, Bologna, Italy

${ }^{3}$ Department of Physics, University of Oslo, N-0316 Oslo, Norway

${ }^{4}$ European Commission JRC, Institute for Reference Materials and Measurements, B-244 Geel, Belgium

${ }^{5}$ Johann-Wolfgang-Goethe Universität, Frankfurt, Germany

${ }^{6}$ Uniwersytet Łódzki, Lodz, Poland

${ }^{7}$ Centre National de la Recherche Scientifique/IN2P3 - IPN, Orsay, France

${ }^{8}$ Istituto Nazionale di Fisica Nucleare, Bari, Italy

${ }^{9}$ Centro de Investigaciones Energeticas Medioambientales y Tecnológicas (CIEMAT), Madrid, Spain

${ }^{10}$ Charles University, Prague, Czech Republic

${ }^{11}$ Commissariat à l'Énergie Atomique (CEA) Saclay - Irfu, Gif-sur-Yvette, France

${ }^{12}$ European Organization for Nuclear Research (CERN), Geneva, Switzerland

${ }^{13}$ University of Manchester, Oxford Road, Manchester, UK

${ }^{14}$ Department of Physics, Faculty of Science, University of Zagreb, Croatia

${ }^{15}$ Universitat Politecnica de Catalunya, Barcelona, Spain

${ }^{16}$ Instituto Tecnológico e Nuclear, Instituto Superior Técnico, Universidade Técnica de Lisboa, Lisboa, Portugal

${ }^{17}$ Universidad de Sevilla, Spain

${ }^{18}$ National Technical University of Athens (NTUA), Greece

${ }^{19}$ Instituto de Física Corpuscular, CSIC-Universidad de Valencia, Spain

\footnotetext{
ae-mail: massimi@bo.infn.it
} 
${ }^{20}$ Universidade de Santiago de Compostela, Spain

${ }^{21}$ Paul Scherrer Institut, Villigen PSI, Switzerland

${ }^{22}$ Aristotle University of Thessaloniki, Thessaloniki, Greece

${ }^{23}$ Bhabha Atomic Research Centre (BARC), Mumbai, India

${ }^{24}$ Atominstitut, Technische Universität Wien, Austria

${ }^{25}$ University of York, Heslington, York, UK

${ }^{26}$ Karlsruhe Institute of Technology, Campus Nord, Institut für Kernphysik, Karlsruhe, Germany

${ }^{27}$ University of Vienna, Faculty of Physics, Austria

${ }^{28}$ Istituto Nazionale di Fisica Nucleare, Laboratori Nazionali di Legnaro, Italy

${ }^{29}$ Agenzia nazionale per le nuove tecnologie, l'energia e lo sviluppo economico sostenibile (ENEA), Bologna, Italy

${ }^{30}$ Istituto Nazionale di Fisica Nucleare, Laboratori Nazionali di Trieste, Italy

${ }^{31}$ Horia Hulubei National Institute of Physics and Nuclear Engineering - IFIN HH, Bucharest - Magurele, Romania

${ }^{32}$ Department of Physics and Astronomy - University of Basel, Basel, Switzerland

\begin{abstract}
We have measured the radiative neutron-capture cross section and the total neutron-induced cross section of one of the most important isotopes for the s process, the ${ }^{25} \mathrm{Mg}$. The measurements have been carried out at the neutron time-of-flight facilities n_TOF at CERN (Switzerland) and GELINA installed at the EC-JRC-IRMM (Belgium). The cross sections as a function of neutron energy have been measured up to approximately $300 \mathrm{keV}$, covering the energy region of interest to the s process. The data analysis is ongoing and preliminary results show the potential relevance for the s process.
\end{abstract}

\title{
1 Introduction
}

The slow neutron capture process (s process) in stars is responsible for the production of about half of the elemental abundances beyond iron that we observe today [1,2]. Most of the s-process isotopes between iron and strontium $(60<\mathrm{A}<90)$ are produced in massive stars [3] $\left(\mathrm{M}>10-12 \mathrm{M}_{\text {sun }}\right)$ where the ${ }^{22} \mathrm{Ne}(\alpha, \mathrm{n})^{25} \mathrm{Mg}$ reaction is the main neutron source. Beyond strontium, the s-process abundances are mostly produced in low mass Asymptotic Giant Branch stars [4] (1.2 $\mathrm{M}_{\text {sun }}<\mathrm{M}<3 \mathrm{M}_{\text {sun }}$ ), where the neutrons are provided by the ${ }^{13} \mathrm{C}(\alpha, \mathrm{n}){ }^{16} \mathrm{O}$ reaction and by the partial activation of the ${ }^{22} \mathrm{Ne}(\alpha, \mathrm{n}){ }^{25} \mathrm{Mg}$ reaction. In stars with an initial metal content similar to solar, ${ }^{25} \mathrm{Mg}$ is the most important neutron poison via neutron capture on ${ }^{25} \mathrm{Mg}$ in competition with neutron capture on ${ }^{56} \mathrm{Fe}$ that is the basic s-process seed for the production of the heavier isotopes. For this reason, a precise knowledge of the ${ }^{25} \mathrm{Mg}(\mathrm{n}, \gamma)^{26} \mathrm{Mg}$ is required to properly simulate s-process nucleosynthesis in stars.

In addition the ${ }^{25} \mathrm{Mg}(\mathrm{n}, \gamma)^{26} \mathrm{Mg}$ reaction cross-section gives important constraints for the yet uncertain reaction rate of the important neutron source ${ }^{22} \mathrm{Ne}(\alpha, \mathrm{n})^{25} \mathrm{Mg}$. The relevant information in this respect are the spin and parity of the neutron resonances formed in the (n, $\gamma$ ) and in the (n, tot) reaction, which can be deduced from a combined resonance shape analysis of neutron capture and total cross section data.

Taking advantage of the features of the neutron time-of-flight (TOF) facilities $\mathrm{n}_{-}$TOF [5] at CERN, and GELINA [6] at IRMM, i.e. the high energy resolution and low background, capture measurements have been performed in 2003 [7] and - with an improved experimental setup - in 2012 at n_TOF and a transmission measurement has been carried out at GELINA. 


\section{Experimental setup}

The TOF technique enables the measurement of energy dependent reaction cross-sections, in particular both $\mathrm{n}$ _TOF and GELINA cover a wide energy range from thermal to the $\mathrm{GeV}$ and from thermal to the $\mathrm{MeV}$ neutron energy, respectively. The (n, $\gamma$ ) experiments have been carried out at the 185-m measurement station of the $n \_$TOF facility, while the (n, tot) measurement was performed at a 50-m measurement station of the GELINA facility.

\section{$2.1 \mathrm{n}$ _TOF installation and its measurement instrumentation}

The $n \_$TOF facility is a white neutron source, where neutrons are produced by spallation of a 20$\mathrm{GeV}$ proton beam on a massive lead block. More details can be found in Ref. [5]. In the new setup, the initially-fast neutrons are moderated by a $5-\mathrm{cm}$ Borated water layer, surrounding the neutronproducing target.

Capture events (i.e. the prompt $\gamma$-rays from the de-excitation of compound nucleus) from the magnesium sample were recorded using the total energy method based on $\mathrm{C}_{6} \mathrm{D}_{6}$ detectors and applying the pulse height weighting technique (PHWT) (see Ref. [7] and references therein). Two deuterated benzene liquid scintillators were used in the measurement. They consisted of cylindrical cells with an active volume of about 1000 and $750 \mathrm{~cm}^{3}$, respectively. Their position was perpendicular to the beam, $9.2 \mathrm{~cm}$ upstream from the sample center in order to reduce the background due to scattered photons.

The neutron fluence, at the sample position, was determined by combining data from several flux detectors based on the ${ }^{6} \mathrm{Li}(\mathrm{n}, \alpha){ }^{3} \mathrm{H},{ }^{10} \mathrm{~B}(\mathrm{n}, \alpha){ }^{7} \mathrm{Li}$, and ${ }^{235} \mathrm{U}(\mathrm{n}, \mathrm{f})$ standard cross sections as explained in Ref. [5].

The experimental observable is the capture yield $Y$, which is in first approximation related to the radiative capture cross-section $\sigma_{\gamma}$ and the areal density of the sample $n$ by: $Y(E) \approx n \times \sigma_{\gamma}(E), E$ being the neutron energy.

\subsection{The GELINA installation and its measurement instrumentation}

The GELINA facility is a multi-user facility with 10 measurement stations. Neutrons are produced by $100-\mathrm{MeV}$ electrons impinging on a Uranium target, via Bremsstrahlung, i.e. by $(\gamma, \mathrm{n})$ and $(\gamma, \mathrm{f})$ reactions. To produce a white neutron spectrum from thermal energy up to a few MeV, two $4 \mathrm{~cm}$ thick beryllium containers filled with water, placed beneath and above the target, are used as moderators.

In the transmission experiment, the neutron beam passing through the sample was collimated to reduce its diameter to less than $35 \mathrm{~mm}$ at the sample position. The sample was placed in an automatic sample changer at a distance of $23 \mathrm{~m}$ from the neutron source. The neutrons were detected by a Li-glass scintillator enriched to $95 \%$ in ${ }^{6} \mathrm{Li}$ and connected through a boron-free quartz window to a 127 mm EMI 9823 KQB photomultiplier (PMT) see Ref. [8] for more details.

The experimental observable is the transmission $T$, defined as the ratio between the neutron flux passing through the sample without interacting and the flux impinging on the sample. This observable is in first approximation related to the total cross-section $\sigma_{\text {tot }}$ and the areal density of the sample by: $\ln T(E) \approx-n \times \sigma_{\text {tot }}(E)$.

\section{Preliminary results and conclusion}

The Mg samples used in both experiments are metal discs, with an enrichment in ${ }^{25} \mathrm{Mg}$ of $98 \%$ (the natural abundance is $10 \%$ ). The use of a metal sample avoids the drawbacks of oxide powder (accumulation of moisture and inhomogeneities) and minimizes the background due to the sample holder. 
Table 1. Sample characteristics and their use in the experiments.

\begin{tabular}{ccccc}
\hline mass & diameter & thickness & area density & type of \\
\hline $3.943 \pm 0.002 \mathrm{~g}$ & $20 \mathrm{~mm}$ & $\approx 0.66 \mathrm{~cm}$ & $3.03 \times 10^{-2} \mathrm{at} / \mathrm{b}$ & Capture \\
$25.102 \pm 0.002 \mathrm{~g}$ & $36 \mathrm{~mm}$ & $\approx 1.19 \mathrm{~cm}$ & $5.95 \times 10^{-2} \mathrm{at} / \mathrm{b}$ & Transmission \\
\hline \hline
\end{tabular}

Details of the sample, which was provided by the National Isotope Development Center of the Oak Ridge National Laboratory - USA, are listed in table 1. In the left panel of Fig. 1, the capture data collected in 2003 are compared with the recent measurement in 2012. The new and more accurate capture measurement has the advantage of using a pure sample without contaminants, in addition the uncertainty on the mass of the sample is very small, therefore the uncertainty on capture cross-section is expected to be drastically reduced with respect to Ref. [7]. In the right panel of Fig. 1 the transmission data from this work is compared with the existing data from a previous measurement [9], where a natural $\mathrm{Mg}$ sample was used. In the present measurement the $\mathrm{n}+{ }^{25} \mathrm{Mg}$ resonances can be identified.
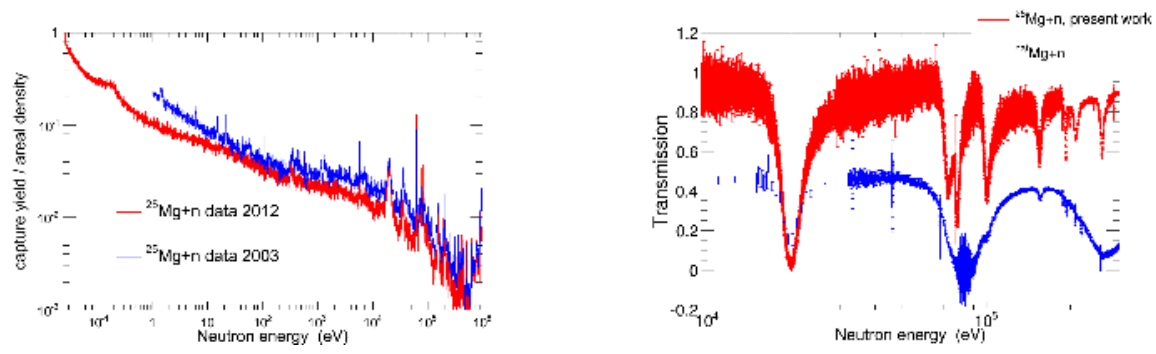

Figure 1. Left panel: capture data obtained at $\mathrm{n}_{-} \mathrm{TOF}$ with different ${ }^{25} \mathrm{Mg}$ samples and experimental setup. The 2012 capture measurement (in red) clearly shows a reduced background. Right panel: Transmission data obtained at GELINA (in red) compared with a previous measurement, where a natural Mg sample was used.

To conclude, the combined results of these measurements on ${ }^{25} \mathrm{Mg}$, are providing the required accuracy for a substantially improved discussion of the astrophysical implications for the s process.

\section{References}

[1] E. M. Burbidge, G.R. Burbidge, W.A. Fowler, F. Hoyle , Rev. Mod. Phys. 29, 547 (1957)

[2] F. Käppeler, R. Gallino, S. Bisterzo, and Wako Aoki, Rev. Mod. Phys. 83, 157 (2011)

[3] S.E. Woosely, A. Heger, and T.A. Weaver, Rev. Mod. Phys. 74, 1015 (2002)

[4] M. Busso, R. Gallino, G. Wasserburg, Ann. Rev. Astron. Astrophys. 37239 (1999)

[5] C. Guerrero, et al., The European Physical Journal A 49, 27 (2013)

[6] I. Sirakov, B. Becker, R. Capote, E. Dupont, S. Kopecky, C. Massimi, P. Schillebeeckx, The European Physical Journal A 49, 144 (2013)

[7] C. Massimi, et al., Phys. Rev. C 85044615 (2012)

[8] P. Schillebeeckx, B. Becker, Y. Danon, K. Guber, H. Harada, J. Heyse, A.R. Junghans, S. Kopecky, C. Massimi, M. Moxon, N. Otuka, I. Sirakov, and K. Volev, Nuclear Data Sheets 113, 3054 (2012)

[9] H. Weigmann, R.L. Macklin, and J.A. Harvey, Phys. Rev. C 14, 1328 (1976) 